\title{
The Importance of Quality Control for Clinical PET Imaging
}

\author{
Joshua D. Schaefferkoetter ${ }^{1}$, Medhat Osman ${ }^{2}$, and David W. Townsend ${ }^{1}$ \\ ${ }^{1}$ ASTAR-NUS Clinical Imaging Research Centre, Singapore; and ${ }^{2}$ Department of Nuclear Medicine, Saint Louis University, \\ St. Louis, Missouri
}

$\mathbf{M}$ olecular imaging is uniquely positioned to address the challenges presented by precision medicine, not only in accurately staging disease but also in assessing targeted therapies, thereby potentially improving outcome. This is already being realized with the recent growth in theranostics, where PET plays a role in both the diagnostic and the therapy monitoring stages. As the number of pairs of diagnostic and therapeutic agents increases, PET will continue to play a major role in such precision medicine, at least to the extent to which the requirement for quantitative accuracy is met.

In fact, since its inception back in the 1950s, PET has always been a quantitative measurement technique. After the administration of a radiolabeled compound, PET imaging procedures acquire the time course of the absolute radiotracer concentration for each volumetric element (voxel) within the field of view. Compartmental models can be used, with measurements of the blood activity and the temporal variation of the radioactive concentration in tissue, to estimate the underlying tracer kinetics in each voxel of the imaged volume. Early work in the 1970s established PET as a quantitative technique for making accurate physiologic measurements of cerebral blood flow with ${ }^{15} \mathrm{O}$ and glucose utilization with ${ }^{18} \mathrm{~F}-\mathrm{FDG}$, and fatty acid metabolism in the myocardium with ${ }^{11} \mathrm{C}$-palmitate.

However, the role of PET as a technique to measure human physiology began to change during the 1990s. The realization that ${ }^{18} \mathrm{~F}$-FDG PET could potentially identify regions of abnormal glucose metabolism associated with the presence of a tumor presented a powerful application in oncology for the technique. When financial reimbursement became possible for certain oncologic studies, PET was established as a clinical imaging tool to stage malignant disease. Here, clinical PET diverged from the rigorous quantitative imaging technique that had been introduced in the 1980s. Instead of providing a full, dynamic acquisition, clinical PET provided a single static snapshot of glucose uptake, acquired around 60 min after radiotracer injection.

Received Jun. 30, 2017; revision accepted Sep. 13, 2017.

For correspondence or reprints contact: Medhat M. Osman, Saint Louis University, Saint Louis University Hospital, 3635 Vista Ave., FDT-2, St. Louis, MO 63110.

E-mail: mosman@slu.edu

Published online Oct. 17, 2017.

COPYRIGHT (c) 2017 by the Society of Nuclear Medicine and Molecular Imaging. DOI: $10.2967 /$ jnmt.117.198465

The acquired images were then reviewed to identify regions of abnormal uptake that could indicate cancer.

This divergence from full quantitation continued when the introduction of PET/CT in 2001 opened up the possibility of significantly shorter scan times and increased patient throughput, which, together with expanding reimbursable applications, led to the rapid clinical adoption of the technology. Throughout this process, most clinical PET/CT images were reviewed visually (qualitatively) for regions of abnormal tracer uptake. The definition of "abnormal" was based on the perceived level of tracer uptake in a region of interest. A semiquantitative approach was introduced based on an SUV, a dimensionless number, used to normalize the radiotracer uptake in the image between different patients, scanners, and scan protocols. SUV is defined as the tracer concentration in a region of interest delineating the tumor in absolute units $(\mathrm{MBq} / \mathrm{mL})$ divided by the injected dose per unit body weight $(\mathrm{MBq} / \mathrm{g})$. The injected dose is decaycorrected to the time of image acquisition, and it is assumed that the entire dose is injected intravenously into the patient as a bolus. An arbitrary threshold is often applied to the interpretation of the image such that an SUV below the threshold suggests a benign process whereas an SUV above the threshold is likely indicative of cancer. Even though numerous factors can affect the SUV calculation, many physicians systematically report an SUV in association with each lesion. With comparisons between baseline SUVs and posttreatment SUVs increasingly being used for monitoring therapy and assessing response, the accuracy of the SUV estimate has become an important issue.

In a recent publication, Kinahan et al. suggested that, to meet the demands of precision medicine and achieve an acceptable level of accuracy for an SUV to assess treatment response, the entire imaging process needed quality assurance and quality control (1). Although efforts exist to calibrate equipment, standardize imaging protocols, and minimize patient preparation differences, less attention has generally been paid to the quality of the administration of the radiotracer. This is because, up till now, there has been no effective way to monitor the quality of the injection process; this is particularly significant given that the category of patients requiring PET/CT scans for oncology often have compromised circulatory systems. Furthermore, the accuracy of the SUV 
estimate should be independent of the experience, skill, training, and technique of the technologist.

For a variety of reasons, the quality of the injection process has received little attention in the literature and is usually taken for granted. Only 5 studies from 3 centers have been published addressing this issue. When the activity is not injected cleanly as a bolus, the supply of ${ }^{18} \mathrm{~F}-\mathrm{FDG}$ to the tissue is affected in an unknown way. Since the SUV reflects tumor uptake assuming a bolus injection, any variation in the supply of ${ }^{18} \mathrm{~F}-\mathrm{FDG}$ will directly impact the numeric value of the SUV. For example, if the injected activity infiltrates the extravascular space, the supply to the tumor will be reduced or delayed, and the assumption of a bolus administration may result in an underestimation of the SUV by an unknown amount, potentially reducing image quality and affecting patient management. This is a significant consideration, especially for longitudinal studies, where a comparison of SUVs acquired before and after therapy based on different injection profiles may alter patient management.

In this issue of Journal of Nuclear Medicine Technology, Muzaffar et al. report on the use of a novel quality assurance device that monitors time-activity curves from the patient during the uptake period after injection of the ${ }^{18}$ F-FDG (2). Such curves characterize the injection process, independently of whether the injection site is imaged during the subsequent scanning. In fact, even though it is increasingly unlikely that the injection site will be imaged, such information may not be indicative of what happened during the injection, or even during the subsequent uptake period. The device described by Muzaffar et al. can provide quality control for each injection, and the acquired time-activity curves offer insight to the radiologist or nuclear medicine physician as to the accuracy of the SUV estimate they may have to use to make a patient management decision. Obviously, whereas radiotracer administration problems can never be completely eliminated, an appropriate quality assurance protocol coupled with continuous feedback to the technologists should help minimize such problems, as has been reported in the study.

Other studies for which such a quality control procedure could be important include myocardial perfusion imaging, which is the most commonly performed nuclear medicine study. Radionuclide infiltration at either the rest or stress parts of the examination may lead directly to an incorrect interpretation of the study, with serious consequences for patient management. It is worth noting that for a myocardial perfusion imaging study the injection site is routinely outside the imaging field of view. Also, as mentioned previously, the growth in theranostics implies an increasing number of treatments requiring the injection of therapeutic levels of radioactivity, with serious implications if the dose administration is compromised in any way.

As health care enters the era of precision medicine there will be increasing demands on the quantitative accuracy of molecular imaging techniques. As defined by the National Institutes of Health, precision medicine is "an emerging approach for disease treatment and prevention that takes into account individual variability in genes, environment, and lifestyle for each person" (3). However, to complement the data from genomics (DNA), proteomics (amino acids), and metabolomics (small-molecule metabolites), molecular imaging must offer detailed multiparametric and multimodality information. This implies a return to that past era when PET was considered a quantitative measurement technique of human physiology in vivo. This will also demand a more quantitative PET methodology beyond a simple SUV and require higher quality standards in all aspects of imaging procedures.

However, as is well known, if a return to more quantitative PET procedures is required, a full kinetic analysis of dynamic PET data implies a continuous measurement of radioactivity in the arterial blood, termed the arterial input function. This is one drawback to performing fully quantitative PET studies clinically because placement of an arterial catheter requires special training and many patients would be unwilling to undergo the procedure. In some cases, it may be possible to extract the arterial input function from the images when a major vessel is located in the field of view, but an external sensor monitoring arterial activity noninvasively could be a major step in bringing fully quantitative PET to a clinical reality. Just as for the simplified SUV analysis, where knowledge of the venous injection profile is important, fully quantitative studies require knowledge of the arterial activity throughout the time frame of the image acquisition. Nevertheless, for many reasons, it may never be practical to perform fully quantitative PET for all clinical studies, and the simple SUV approach will likely continue to play a role in staging malignant disease and monitoring therapy. However, in many situations, fully quantitative PET studies may be necessary in the validation stages of a more simplified analysis. Quantitation, and the accompanying quality control procedures discussed here, will be increasingly important as precision medicine becomes a reality, with targeted therapies applied to individual patients rather than members of a heterogeneous group. In all cases, including the growing field of theranostics, quality control and quality assurance of the injection and scanning protocol will play an important role in ensuring that the numbers are meaningful.

\section{REFERENCES}

1. Kinahan PE, Mankoff DA, Linden HM. The value of establishing the quantitative accuracy of PET/CT imaging. J Nucl Med. 2015;56:1133-1134.

2. Muzaffar R, Frye SA, McMunn A, Ryan K, Lattanze R, Osman MM. Novel method to detect and characterize ${ }^{18} \mathrm{~F}-\mathrm{FDG}$ infiltration at the injection site: a single-institution experience. J Nucl Med Technol. 2017;45:267-271.

3. What is precision medicine? Genetics Home Reference website. https://ghr.nlm. nih.gov/primer/precisionmedicine/definition. Published October 24, 2017. Accessed October 26, 2017. 